\title{
Teixobactin: A Powerful Tool for Combating Resistant Strains
}

\author{
TEJAL RAWAL* AND SHITAL BUTANI \\ Department of Pharmaceutics, Institute of Pharmacy, Nirma University, Ahmedabad-382 481, India
}

\section{Rawal and Butani: Teixobactin against Drug-resistant Pathogens}

\begin{abstract}
Resistance to antibiotics has grown out to be a serious health concern. Despite this serious health crisis, no new antibiotics have been discovered since the last 30 years. A new ray of hope in the form of teixobactin has come out of the dark, which could demonstrate the potential to be effective in countering resistance. This new antibiotic has an interesting mechanism of action against bacteria. The discovery of this wonderful compound has evolved as a major breakthrough especially in this era of antibiotic catastrophe. This review article highlights various facets of teixobactin that include chemistry, mode of action, in vitro and in vivo activities. Though the compound has not yet undergone clinical studies, its effect on mice models has given hope for overpowering resistance. This review attempts to provide information about teixobactin and its potential for fighting as antibiotic resistance.
\end{abstract}

Key words: Teixobactin, antibiotic, Eleftheria terrae, iChip, resistance, antimicrobial

A new crisis the world facing today is antibiotic resistance. Genetic modifications in bacteria have led to a condition, where pathogenic microorganisms have become more virulent and resistant to the available antimicrobial agents. The risk of resistance has also been accelerated due to overuse of the antibiotics. The most common example of overuse is by the people suffering from common cold and cough using antibiotics contributing to the development of antimicrobial resistance ${ }^{[1-6]}$.

In recent years, pharmaceutical companies have reduced research efforts to develop new antibiotics. During 1980s, a new antibiotic teixobactin was discovered, which has potential to overpower many bacteria. Though this antibiotic might not be effective against some highly dreadful bugs, but it has the potential to make a huge influence on public health. However, this antibiotic still needs to undergo clinical trials. An important point to be considered in case of teixobactin is that it is active only against Grampositive bacteria. Teixobactin has not been reported to be effective in Gram-negative bacteria. The major reason for this might be the presence of an extracellular membrane in Gram-negative bacteria which protects them from several chemicals ${ }^{[7-14]}$.

In the past, the well-known antibiotic, penicillin, which gained the title of 'remarkable drug' as well as referred to as "magic bullet" by Paul Ehlrich, gained these recognitions since it was highly effective against bacteria, without causing any harm to the body. It was found to be effective against organisms, where sulphonamides failed. Alexander Fleming who discovered penicillin warned about the development of resistance to the super drug. Later on, several resistant strains were unearthed. Fleming's words served as an alarm to the world about the fact that the main cause of antibiotic resistance is the misuse of these drugs. Though the discovery of penicillin paved the path to discover more and more antibiotics, but resistance remained as a major challenge. This menace is driving the scientists to explore newer antibiotics, which can combat the resistant strains. In this struggle, teixobactin has evolved as a ray of hope for dealing with resistant bacteria $^{[15]}$.

There exists a desperate need for new antibiotics since the resistant strains have evolved as the major

This is an open access article distributed under the terms of the Creative Commons Attribution-NonCommercial-ShareAlike 3.0 License, which allows others to remix, tweak, and build upon the work non-commercially, as long as the author is credited and the new creations are licensed under the identical terms

Accepted 15 November 2016

Revised 17 October 2016

Received 02 July 2016

Indian J Pharm Sci 2016;78(6): 697-700 
cause of suffering. Since long, no new antibiotic has been uncovered making the antimicrobial resistance a major concern. This alarming situation has raised the call for newer options in antibiotics. Scientists are struggling to discover new antibiotics since many years for countering the antibiotic resistance. One approach which has proved to be fruitful since many years is the discovery of new molecules through soil bacteria. After so many attempts, finally the scientists have succeeded in isolating an antibiotic, which exhibited potential against resistant strains and this antibiotic is teixobactin ${ }^{[16-21]}$.

Teixobactin is basically a macrocyclic depsipeptide, which consist of $4 \mathrm{D}$-amino acids, methylphenylalanine and enduracididine ${ }^{[20]}$. It is composed of 11 amino acid chain, which is linked by about 10 peptide linkages. The last four linkages form a square shape. It consists of following amino acids, alanine, threonine, enduracididine, 4-isoleucine moieties, D-glutamine, 2 L-serine moieties and N-methyl-D-phenylalanine ${ }^{[22]}$.

Many antibiotics have come out of the loam. For example, penicillin was obtained from a soil fungus named Penicillium and vancomycin also came from the soil bacteria. Similarly, teixobactin comes from a soil microbe named Eleftheria terrae. A device called iChip (a device consisting of specially designed 96 chambers, each surrounded by a semipermeable membrane) had been used by scientists in which the soil samples diluted with agar was placed. Then the iChip was immersed in the soil which allowed bacterial growth due to diffusion of numerous growth factors and nutrients (fig. 1). This method can be used in future for the development of several more potent antibiotics. After screening 10000 strains of bacteria, 25 potential compounds were selected of which one is teixobactin with potent activity against Gram-positive bacteria $^{[23-26]}$.

Teixobactin acts by interfering with the synthesis of cell wall due to its ability to bind to two lipids, lipid II, which is a peptidoglycan precursor and lipid III, which is a teichoic acid (component of Gram-positive bacterial cell wall) precursor (fig. 2). It mainly attacks cell wall instead of targeting proteins. So basically, teixobactin can be considered as an inhibitor of peptidoglycan synthesis with practically no effect on

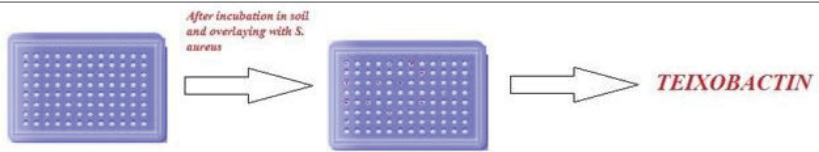

Fig. 1: Discovery of teixobactin using iChip.

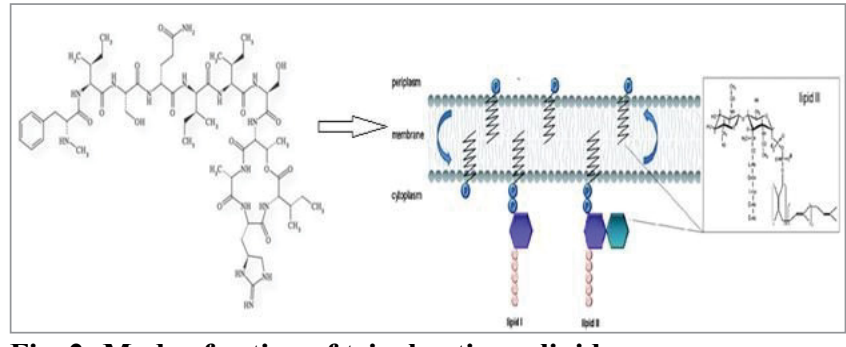

Fig. 2: Mode of action of teixobactin on lipids.

the proteins, DNA or RNA. During the past 3 decades, it is the first new potent antibiotic reported. In mice models, it has shown potent activity against several resistant bacterial strains such as of Streptococcus pneumonia and Mycobacterium tuberculosis. It was also found to be active against methicillin-resistant $S$. aureus infections. It prevented the build-up of outer coats of the bacteria ${ }^{[20,21,26]}$. It appeared to have effect against some drug-resistant bacterial strains for example, methicillin-resistant $S$. aureus (MRSA) and vancomycin-resistant enterococci (VRE). It can also kill Clostridium difficile, M. tuberculosis and has the potential to be used as a treatment for tuberculosis (Table 1) $)^{[22,27,28]}$.

Teixobactin basically contains two massive nonribosomal peptide synthetase (NRPS)-coding genes named txo1 and txo2. When the sequencing of genome of E. terrae was done, it was found on the basis of $16 \mathrm{~S}$ rDNA and in silico hybridization of deoxyribonucleic acid that the microorganism belongs to aqua bacteria group (Gram-negative group). A compound with 1242 Da molecular mass was isolated and was studied by mass spectrometry. Further, the compound's stereochemistry was explored using nuclear magnetic resonance (NMR) and a homology search was performed to identify the gene cluster. The compound identified was named as teixobactin $^{[21,22]}$.

Teixobactin has shown remarkable activity against several infections and is under preclinical development. Researchers at the North Eastern University, Antimicrobial Discovery Centre reported that mice administered with teixobactin survived and did not show any sign of toxicity even after fatal doses of $S$. pneumoniae or MRSA. This prompted the scientists to progress this drug further to preclinical trials. If teixobactin becomes a success in the market, it will prove to be the first novel class of antibiotic in itself ${ }^{[28]}$.

In in vitro experiments, teixobactin was found to be effective in retarding the growth of several Grampositive bacterial strains along with many drugresistant strains. When $S$. aureus was cultured with 
www.ijpsonline.com

TABLE 1: ANTIBIOTIC ACTIVITY AGAINST DIFFERENT MICROORGANISMS

\begin{tabular}{llll}
\hline Drug & Microorganisms & Class of bacteria & Activity \\
\hline Methicillin & S. aureus & Gram-positive & Development of resistance \\
Vancomycin & Enterococci & Gram-positive & Development of resistance \\
Rifampicin & M. tuberculosis & Gram-positive & Development of resistance \\
Teixobactin & S. aureus & Gram-positive & No resistant strains found \\
& Enterococci & Gram-positive & No resistant strains found \\
& M. tuberculosis & Gram-positive & No resistant strains found \\
& B. anthrasis & Gram-positive & No resistant strains found \\
& & All Gram-negative bacteria & Development of resistance \\
\hline
\end{tabular}

low teixobactin levels, no resistance was found to be developed in bacteria. In mouse models, the protective dose for teixobactin and vancomycin were found to be $0.2 \mathrm{mg} / \mathrm{kg}$ and $2.75 \mathrm{mg} / \mathrm{kg}$ for MRSA septicaemia, respectively. The protective dose here refers to the dose of the drug at which half of the animals survived. Thus, we can say that teixobactin is more potent than vancomycin. Reduction with teixobactin in bacterial levels was found to be $26 \mathrm{~h}$ post MRSA infection and $48 \mathrm{~h}$ post $S$. pneumoniae infection.

Ling et al. has shown in their studies that teixobactin was highly effective against Bacillus anthracis and $C$. difficile with minimum inhibitory concentration (MIC) of 5 and $20 \mathrm{mg} / \mathrm{ml}$. No resistance was found in case of M. tuberculosis or $S$. aureus when plating with low dose of teixobactin $(4 \times \mathrm{MIC})$ was done. In their studies, they found that teixobactin mainly inhibited peptidoglycan synthesis but has shown no effect on DNA, proteins or RNA thus giving the clue that protein is not its target.

Ling et al. performed in vivo studies in mice models. In serum, the potency of the compound was maintained. As per their studies, the pharmacokinetic parameters were found to be suitable after single injection IV dose of $20 \mathrm{mg} / \mathrm{kg}$ as the serum level was retained above MIC for about $4 \mathrm{~h}$. In mice septicemia model, the mice infected with MRSA when treated with teixobactin intravenously, were found live. S. pneumoniae infected mice also showed a reduction of $6 \log 10$ of colony forming units (CFU) in lungs with teixobactin ${ }^{[21,22]}$.

Vancomycin is a glycopeptide antibiotic. Though vancomycin is a pretty effective antibiotic yet many bacteria like $S$. aureus have been found to develop resistance. Here comes an important role of teixobactin, which has been found to be active against VRE, and modifications in lipid II. The resistant strain had lipid II-D-Ala-D-Lac in place of lipid II-D-Ala-D-Ala arrangement. Teixobactin exhibited an ability to bind to this altered form of lipid II and render it inactive. Due to this amazing feature, teixobactin appeared to have the potential to be effective in infections caused by vancomycin resistant strains ${ }^{[21]}$.

Numerous acyl depsipeptide antibiotics have emerged as a potent class, which in future is likely to play a key role in combating resistance to antibiotics. Examples are enopeptin A, enopeptin B, A54556A and B545556B. These depsipeptides mainly function by activating protease complex in bacteria which leads to degradation of uncontrolled protease which further hinders the cell division ultimately leading to cell death. The mechanism is based on the fact that the acyl depsipeptides cause degradation of protein FtsZ, which played a crucial role in cell division. This protein degradation further leads to failure of Z-ring formation ${ }^{[29]}$.

Enopeptins A and B are acyl depsipeptides, which have been isolated from Streptomyces species RK-1051 from soil sample. These have been found to show high activity against $S$. aureus with a MIC of $12.5 \mu \mathrm{g} / \mathrm{ml}$. Its activity against Gram-negative mutants has also been unveiled. The structure of enopeptin consisted of $\mathrm{z}$ lactone nucleus made up of amino acids and side chain of phenylalanine ${ }^{[30]}$. These have been isolated from a mixture of depsipetide factors from A-H (8 factors) and have been originated from $S$. hawaiiensis. They interfere with normal bacterial cell division which serves as their main mode of action ${ }^{[31]}$.

Since last 30 years, no new antibiotic class has been identified in spite of an urgent requirement for a suitable antibiotic to counter antimicrobial resistance. Teixobactin turned out to be the first new class of antibiotics with considerable activity against resistant strains. This antibiotic has the potential to be a powerful tool to combat resistance microorganisms as has it exhibited excellent activity in mice models.

\section{Conflicts of interest:}

There are no conflicts of interest. 


\section{Financial support and sponsorship:}

Nil.

\section{REFERENCES}

1. Sandle T. Teixobactin: A new class of antibiotic. SOJ Microbiol Infect Dis 2015;3:1-2.

2. http://www.2minutemedicine.com/teixobactin-appears-avoidbacteria-resistance-preclinical/.

3. http://antibiotic-action.com/wp-content/uploads/2015/01/ Piddock-comment-on-new-antibiotic-teixobactin-8-1-15.

4. Piddock LJV. Teixobactin, the first of a new class of antibiotics discovered by iChip technology? J Antimicrob Chemother 2015;70:2679-80.

5. Bialvaei AZ, Samadi KH. Colistin, Mechanisms and Prevalence of Resistance. Curr Med Res Opin 2015;31:1-54.

6. Naik G. Scientists discover potent antibiotic. A potential weapon against a range of diseases. Wall St J 2015.

7. http://www.medicaldaily.com/teixobactin-prevents-antibioticresistance-and-kills-superbugs-will-it-work-humans-317034.

8. http://www.nhs.uk/news/2015/01january/pages/new- gamechanging- antibiotic discovered.aspx.

9. Mishra D. Teixobactin: An antibiotic that can trick bacteria. Business Standard India 2015.

10. Wright G. Antibiotics: An irresistible newcomer. Nature 2015;517:442-4.

11. Healy M. Teixobactin and the hope it represents for fighting antibiotic resistance. Los Angeles Times 2016.

12. Lewis K. NovoBiotic reports the discovery of teixobactin, a new antibiotic without detectable resistance, Cambridge, Massachusetts. Novo Biotic Pharmaceuticals 2015.

13. http://www.theweek.co.uk/world-news/62014/teixobactingame-changing-new-antibiotic-discovered.

14. http://zeenews.india.com/news/health/health-news/newblockbuster-antibiotic-teixobactin-discovered 1526839.

15. Levy SB. The Antibiotic Paradox: How the Misuse of Antibiotics Destroys Their Curative Powers. Da Capo Press 2002.
16. Sample I. New class of antibiotic could turn the tables in battle against superbugs. The Guardian 2015.

17. Ledford H. Promising antibiotic discovered in microbial "dark matter". Nature 2015.

18. Sherritze S. Chipping away at antibiotic resistance, hideous hospital gowns and other health care woes. P T 2015;40:304-5.

19. http://www.lrb.co.uk/blog/2015/01/08/hugh-pennington/ teixobactin/.

20. http://rt.com/usa/221003-ichip-teixobactin-discovery-study/.

21. Ling LL, Schneider T, Peoples AJ, Spoering AL, Engels I, Conlon BP, et al. A new antibiotic kills pathogens without detectable resistance. Nature 2015;517:455-9.

22. http://www.abc.net.au/news/2015-01-09/scientists-discovernew-antibiotic-for-first-time-in-30-years/6007730.

23. Stone J, Judy S. Teixobactin and iChip Promise Hope against Antibiotic Resistance. Forbes 2015.

24. Nichols D, Cahoon N, Trakhtenberg EM, Pham L, Mehta A, Belanger A, et al. Use of ichip for high-throughput in situ cultivation of "uncultivable" microbial species. Appl Environ Microbiol 2010;76:2445-50.

25. Denise G. From a pile of dirt, hope for a powerful new antibiotic. The New York Times 2015.

26. http://www.doomandbloom.net/teixobactin-a-new-antibiotic/.

27. http://sciencemadeeasy.kinja.com/teixobactin- won-t- saveus- all- but- it- s- a- good- start 1683087705.

28. http://www.acs.org/content/acs/en/molecule-of-the-week/ archive/t/teixobactin.html.

29. Lee BG, Park EY, Lee KE, Jeon H, Sung KH, Paulsen H, et al. Structures of ClpP in complex with acyldepsipeptide antibiotics reveal its activation mechanism. Nat Struct Mol Biol 2010;17:471-8.

30. Koshino H, Osada H, Yano T, Uzawa J, Isono K. The structure of enopeptins $\mathrm{A}$ and $\mathrm{B}$, novel depsipeptide antibiotics. Tetrahedron Lett 1991;32:7707-10.

31. Hinzen B, Raddatz S, Paulsen H, Lampe T, Schumacher A, Häbich D, et al. Medicinal chemistry optimization of acyldepsipeptides of the enopeptin class antibiotics. Chem Med Chem 2006;1:689-93. 